\title{
$\widehat{A}$ Madridge \\ madridge Journal of Dermatology \& Research \\ Interconnecting Scientific World
}

Research Article

Open Access

\section{Hair Growth Stimulation by a Natural Remedy: Animal Studies}

\author{
Khava S Vishnyakova ${ }^{1}$, Larisa G Vetkova ${ }^{2}$, Maxim V Jasko', Alexander M Aliper ${ }^{3,4}$, Anton A Buzdin ${ }^{3-5}$, \\ Konstantin V Popov', Anna V Kudryavtseva ${ }^{1}$ and Yegor E Yegorov ${ }^{1,6 *}$ \\ ${ }^{1}$ Engelhardt Institute of Molecular Biology, Russian Academy of Sciences, Moscow, Russia \\ ${ }^{2}$ Gamalei Institute of Epidemiology and Microbiology, Ministry of Health of the Russian Federation, Moscow, Russia \\ ${ }^{3}$ Rogachev Center of Pediatric Hematology, Oncology, and Immunology, Ministry of Health of the Russian Federation, Moscow, Russia \\ ${ }^{4}$ Pathwaypharmaceuticals, Ltd., Wan Chai, Hong Kong SAR \\ ${ }^{5}$ Shemyakin and Ovchinnikov Institute of Bioorganic Chemistry, Russian Academy of Sciences, Moscow, Russia \\ ${ }^{6}$ Moscow Institute of Physics and Technology, Ministry of Education and Science of the Russian Federation, Dolgoprudny, Moscow \\ Region, Russia
}

\section{Article Info}

*Corresponding author:
Yegor E Yegorov
Engelhardt Institute of Molecular Biology
Moscow
Russia
E-mail: yegorov58@gmail.com

Received: January 15, 2018

Accepted: January 31, 2018

Published: February 6, 2018

Citation: Vishnyakova KS, Vetkova LG, Jasko MV, et al. Hair Growth Stimulation by a Natural Remedy: Animal Studies. Madridge J Dermatol Res. 2018; 3(1): 37-44. doi: $10.18689 / \mathrm{mjdr}-1000109$

Copyright: @ $₫ 2018$ The Author(s). This work is licensed under a Creative Commons Attribution 4.0 International License, which permits unrestricted use, distribution, and reproduction in any medium, provided the original work is properly cited.

Published by Madridge Publishers

\begin{abstract}
Hair follicles normally regenerate cyclically and this makes them a popular model for investigations of regeneration and aging. We studied the effects on mouse hair follicles of the Satura ${ }^{\oplus}$ Rosta remedy manufactured from plant extracts and used to stimulate hair growth restoration. A prolonged application of the remedy to the skin of various strains of mice and of outbred white mice did not induce detectable negative effects and caused a significant increase of mean hair follicle size and hair root volume as well as skin thickening due to adipose elaboration. The volume increase took place mainly among the largest hair follicles. Mouse skin thickness is known to fluctuate cyclically in coordination with the waves of hair growth; anagen regions are thicker due to adipose increase. Satura ${ }^{\oplus}$ Rosta did not abrogate hair growth waves. Apparently the observed effects could be explained by the remedy-induced prolongation of the anagen phase. Although the effect of Satura ${ }^{\oplus}$ Rosta on fur appearance was insignificant in young animals, experiments with old mice exhibiting alopecia resulted in hair regrowth in alopecia regions. Transcriptome analysis of Satura ${ }^{\circledR}$ Rosta action on dermal fibroblasts in vitro revealed significant changes of the expression of genes associated with the cell cycle, developmental processes and lipid metabolism.
\end{abstract}

Keywords: Hair Growth; Alopecia; Mice; Hair Follicles; Anagen; "Satura ${ }^{\circledR}$ Rosta"; Adipose; Aging; Transcriptome.

\section{Introduction}

The creation of a remedy for effective hair growth stimulation has for many years been a dream of cosmetologists through the entire world. Although alopecia and hair loss are treated by cosmetologists, they are also generally accepted marks of aging. In recent years, there has been a growing interest to hair follicle studies because hair follicle can serve as an informative model for investigation of cell senescence, aging of tissues, organs and the whole body.

Hairs grow cyclically. Several periods can be distinguished within the hair growth cycle. The growing phase of a hair follicle is called anagen, after it occurs catagen-the period of degeneration, and at the end telogen-the rest phase [1]. In human scalp the hair growth cycle spans up to 7 years, in mouse skin-a few weeks. Hair follicles are capable to return to growth after a prolong period of rest. 
There is a great intensity of metabolism in growing follicles. The beginning of the growth is prepared by the accumulation of building materials and energy including the development of subcutis-a thick layer of skin located under the derma and composed mainly of adipocytes. During anagen, the follicles are completely immersed into this adipose layer and enhanced growth of blood capillaries surrounding follicles is observed. In contrast to humans, most of the mice hairs grow synchronously. There are waves of hair growth moving along the body. Waves are strictly oriented in young animals, later the waves became weaker and finally islands of hair growth appear instead of waves. Growth waves cyclically alter skin thickness by influencing adipose elaboration and regression [2]. Skin aging is associated with adipose reduction, decreased cellularity and reduction of metabolism [3]. Age-related skin deterioration should influence hair growth; however hair greying and alopecia develop earlier than classical phenotype of old skin.

There are many types of alopecia that differ in underlying mechanisms and the degree of development. Among etiological factors of alopecia are heredity, autoimmune processes, aging or premature aging, consequences of anticancer therapy and even psychological stress. Pathogenesis of different kinds of alopecia is not completely understood therefore the market offers an impressive number of different medications of which the majorities are ineffective or even have negative side effects.

The most widespread kind of alopecia is the androgenetic one (AGA). It constitutes around $90 \%$ of cases. It is characterized by the appearance of areas of baldness in frontal and parietal zones of man scalp and hair rarefication in the same areas in women. Hair cycles are shortened as a result of diminished anagen that leads to the vellus transformation of scalp hairs, hair follicle miniaturization, and a decrease of hair size up to their complete disappearance [4]. All the hairs in affected areas can be involved in this process. Along with hair miniaturization, the production of pigment ceases. AGA is a very widespread problem: about $50 \%$ of men beyond the age of 50 years and $50 \%$ women beyond the age of 60 years are subject to AGA [5]. Some affected individuals experience great psychoemotional stress, often leading to a reduction of quality of life.

From the early 1940s it has been known that the process of AGA development is mediated mainly by androgen [6]. Disease is characterized by the increased responsiveness of dermal papilla in specific locations to testosterone that leads them to increased secretion of cytokines such as TGF- $\beta$ and DKK-1. TGF- $\beta$ [7-9] is known as an anagen inhibitor in the hair cycle. DKK-1 [10] inhibits the growth of the outer root sheath cells and induces apoptotic cell death. It is likely that these mediators determine an androgen induced inhibition of hair growth and early catagen induction in AGA [11].

There are two US Food and Drug Administrationapproved therapies for treatment of AGA - Minoxidil and Finasteride. Minoxidil is a potassium channel opener, initially used for hypertension treatment. Minoxidil appears to increase the duration of the anagen phase, and its angiogenic effects result in the maintenance of hair follicles sizes [12-14].
Finasteride initially introduced for treatment of benign prostatic hyperplasia selectively inhibits the type 2 $5 \alpha$-reductase isoenzyme responsible for converting testosterone to dihydrotestosterone (DHT), the putative hormonal modulator of AGA in men. Finasteride reduces serum and scalp DHT concentrations by approximately $50 \%$. Finasteride may inhibit or reverse miniaturization of hair follicles. Finasteride is generally well tolerated during a longterm daily application [15-17]. Controversial data exist regarding the therapeutic efficacy of finasteride in treating females AGA [18-20].

Although Minoxidil and Finasteride both retard or stop hair loss and stimulate some hair regrowth, neither medication restores all lost hair or reverses complete baldness. The existing baldness therapies were discovered by accident and precise mechanism of their action are unclear. Hypothetically there are many targets upstream and downstream of androgenic signaling pathway that can be explored for baldness treatment. Other approaches include anti-aging therapies and stimulation of tissue regeneration.

We presume that any anti-aging treatment should have hair stimulating activity. For many years, the balsam "Satura ${ }^{\circledR}$ Rosta" (RSHG) has been known as an effective remedy for baldness treatment. We began to investigate its action and discovered that RSHG can induce adipogenic differentiation [21] and activate autophagy [22]. A few years ago, we began experiments involving long-term application of RSHG to mice. The results of this work are presented here.

\section{Materials and Methods}

\section{Remedy for stimulation of hair growth (RSHG)}

Balsam "Satura ${ }^{\oplus}$ Rosta" (Manufactured by GZ Laboratories (UK) Ltd) is a preparation for baldness treatment. It has been used since 1989 in various countries (USA, Great Britain, Israel, and Russia). This preparation has been tested many times in different laboratories to comply with the safety requirements and sanitary regulations for perfumes and cosmetics. No microorganisms are revealed. Toxic properties comply with the standards and it exhibits no skin irritant or allergenic effects [21]. The exact composition (Intellectual Property and Methodology) of "Satura ${ }^{\circledast}$ Rosta" is wholly owned and trade marked by GZ Laboratories (UK) Ltd. The General Director of GZ Laboratories (UK) Ltd Dr. G.V. Zigmond has advised the authors that the basis of Satura is a processed mixture of numerous terrestrial plant and seaweed extracts.

We used balsam "Satura ${ }^{\circledR}$ Rosta" for testing on animals. For experiments with cell culture we used the basis of the balsam that constitutes only $5 \%$ of the balsam. This basis was provided by Dr. G.V. Zigmond. We prepared a $1 \%$ ethyl alcohol solution and used $0,001 \%$ final concentration.

\section{NMR analysis}

${ }^{1} \mathrm{H}$ NMR spectrum was recorded on an AMX III-400 spectrometer (Bruker BioSpin $\mathrm{GmbH}$, Karlsruhe, Germany) with the working frequency of $400 \mathrm{MHz}$ using DMSO- $\mathrm{d}_{6}$ as the solvent. 


\section{Animal Studies}

The mice were housed in animal quarters of the Gamalei Institute of Epidemiology and Microbiology and received chow ad libitum. All animals were handled in compliance with the Guide for the Care and Use of Laboratory Animals approved by the Institutional Animal Care and Use Committee at the Gamalei Institute of Epidemiology and Microbiology. Mice (Balb/c, CBA, C57BI/6 and outbred white laboratory mice) 2-3 months old were purchased from the Stolbovaya mice hatchery. Initially every experiment included 10 control and 10 experimental mice. Mice were treated with "Satura ${ }^{\circledR}$ Rosta" every second day throughout the experiments. The balsam $(2$ drops $\approx 100 \mu \mathrm{l}$ ) was applied on the back between the shoulders and then spread. The animals were sacrificed at different time points after the beginning of treatment (see Results) and used for immunohistochemistry studies. The obtained skin samples (sites of "Satura ${ }^{\circledR}$ Rosta" applications) were fixed with formalin, embedded in paraffin, and the sections were prepared and stained with hematoxylin-eosin. Plucked hairs were collected at different times (see Results) from the area of balsam application. Hairs were plucked by a single movement to obtain all of them from the same site. The photography was carried out using a Nikon Diavert inverted phase-contrast photomicroscope equipped with a Nikon D5000 camera.

\section{Cell culture}

Primary cultures of human skin fibroblasts (strain 1608) were kindly provided by S.M. Terekhov (Medical Genetic Research Center, Moscow, Russia). Cells were grown in DMEM medium (PanEco, Russia) supplemented with $10 \%$ fetal bovine serum (FBS) (HyClone, USA), 2mM L-glutamine (PanEco, Russia) and $40 \mu \mathrm{g} / \mathrm{ml}$ gentamycin (PanEco, Russia) at $37^{\circ} \mathrm{C}$ in the presence of $5 \% \mathrm{CO}_{2}$. Cells were treated by the $1 \%$ ethyl alcohol solution of the basis of balsam during $24 \mathrm{~h}$. Final concentration of balsam basis was $0,001 \%$. Control cells were treated with solvent only.

\section{RNA isolation, preparation of $m R N A$ libraries and sequencing}

All RNA procedures were described previously [23]. Briefly: samples of cells $(20-30 \mu \mathrm{g})$ frozen in liquid nitrogen were homogenized manually. RNA was isolated using a ZR RNA MiniPrep kit. The RNA amount was estimated using a NanoDrop 1000 spectrophotometer (Nano drop, USA) and a Qubit 2.0 fluorimeter (Invitrogen, USA); RNA quality was checked using an Agilent 2100 bioanalyzer (Agilent Technologies, USA). The RNA integrity number (RIN) was no less than 7 for RNA preparations.

To prepare an mRNA library we used $3.5 \mu \mathrm{g}$ of total RNA isolated as above. Samples were prepared for sequencing with a TruSeqRNA Sample Prep kit v. 2. A total RNA sample $(3.5 \mu \mathrm{g})$ was diluted with $50 \mu \mathrm{L}$ of deionized water. Poly $(A)$ mRNA was isolated with magnetic RNA purification beads (Illumina, USA) coated with poly ( $T$ ). Fragmentation of purified mRNA was also performed at this step. The first cDNA strand was synthesized on the RNA template with Super Script II reverse transcriptase (Invitrogen, United States) and specific primers. The second strand was synthesized using enzymes and primers from the same kit. The resulting dsDNA was purified using AMPure XPBeads (Bekman Coulter, USA). Blunt ends were generated using $3^{\prime}$ - and $5^{\prime}$-exonucleases. A single adenine was added to the blunt $3^{\prime}$ ends to prevent their ligation with each other during ligation of adapters. Ligation to thymine-containing adapters was carried out, and the DNA samples were prepared for hybridization to a flowcell. PCR was run as recommended by the kit manufacturer.

The libraries were tested for quality on an Agilent 2100 analyzer; the concentration was estimated via real-time PCR on a 7500 real-time PCR system (Applied Biosystems, USA). DNA clusters were generated using a cBot device according to an Illumina protocol. The flowcell with DNA clusters obtained using cBot was used for sequencing on an Illumina HiSeq 2000 whole-genome sequencer.

\section{Transcriptome analysis}

A functional analysis of the data was performed using MetaCore software and data-base (GeneGO, Thomson Reuters, and USA). Differential expression was assumed for a gene if its expression was changed (increased or decreased) more than twice compared with the normal level (differential expression threshold $\mid \log 2$ (fold-change)| > 1). A MetaCore functional ontology analysis yielded the MetaCore Maps, MetaCore Networks, and GO Processes that were enriched in differentially expressed genes to the highest extent. To estimate the enrichment significance, $p$-value was calculated using a hypergeometric distribution equation. Biological processes with the lowest $p$-values were considered the most significant. For convenience, we used the negative logarithm (base 10$)$ of the $p$-value ([-log (p-value)]).

\section{Results}

\section{NMR analysis of RSHG}

Multiplet at 3.28 - 3.46 ppm corresponds to signals of glycerol (Fig. 1). The remaining signals indicate the presence of fatty unsaturated acids. Multiplet at 5.30 - 5.32 ppm corresponds to signals $\mathrm{CH}=\mathrm{CH}$ of oleic, linoleic and linolenic acids. The triplet at $2.73 \mathrm{ppm}$ is characteristic for the methylene group $=-\mathrm{CH} 2-=$ of linoleic and linolenic acids. Thus, we see that the basis of RSHG is formed mainly by fatty acid derivatives of glycerol, in which polyunsaturated fatty acids are present.

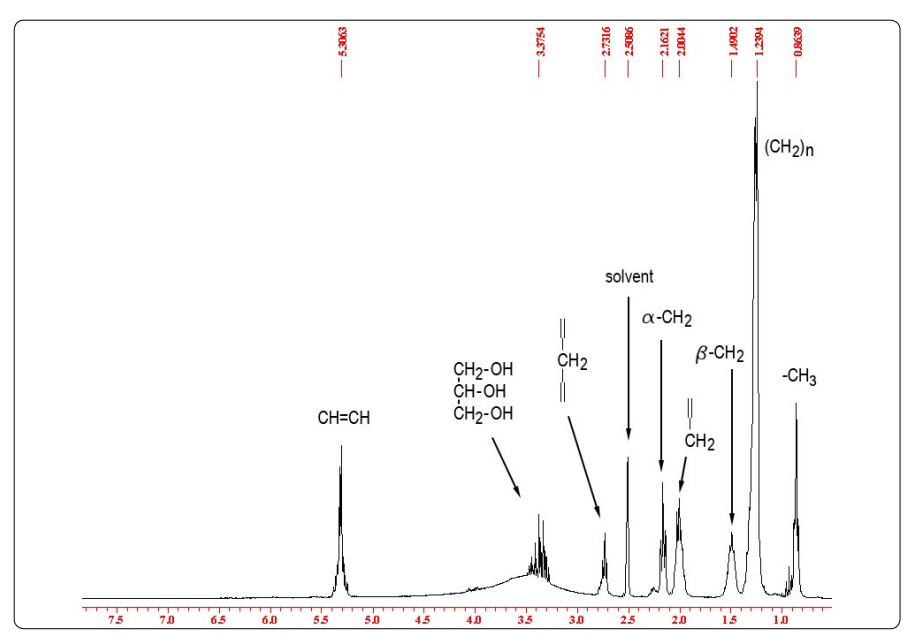

Figure 1. NMR analysis of RSHG 


\section{Long term RSHG application increases hair follicle size}

A series of experiments with continuous RSHG application to the skin was performed. Four kinds of mice were used: Balb/c, CBA, C57BI/6 and outbred white laboratory mice. Groups composed of tens mice each were studied as control and as experimental cohorts. Mice were treated with RSHG on alternate days throughout the experiment. Balsam ( 2 drops $\approx$ $100 \mu \mathrm{l}$ ) was applied on the back between the shoulders and spread. In the process of experiments, the coats of all animals looked well and we could not distinguish between experimental and control animals. Also we did not observe any behavioral peculiarities of experimental mice.

As RSHG is designed to stimulate hair growth, we could suppose that it should increase the hair follicle sizes, so we measured them. For this purpose we searched maximal size follicles on the photos of histological sections. Only one follicle per mouse was included. We sorted these follicles in a descending order (fig.2-3).

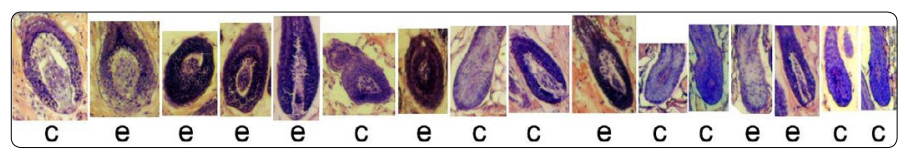

Figure 2. RSHG influence on hair follicles size

This row corresponds to experiment №1 from fig.2. The follicles are sorted in a descending order. c control. e experiment

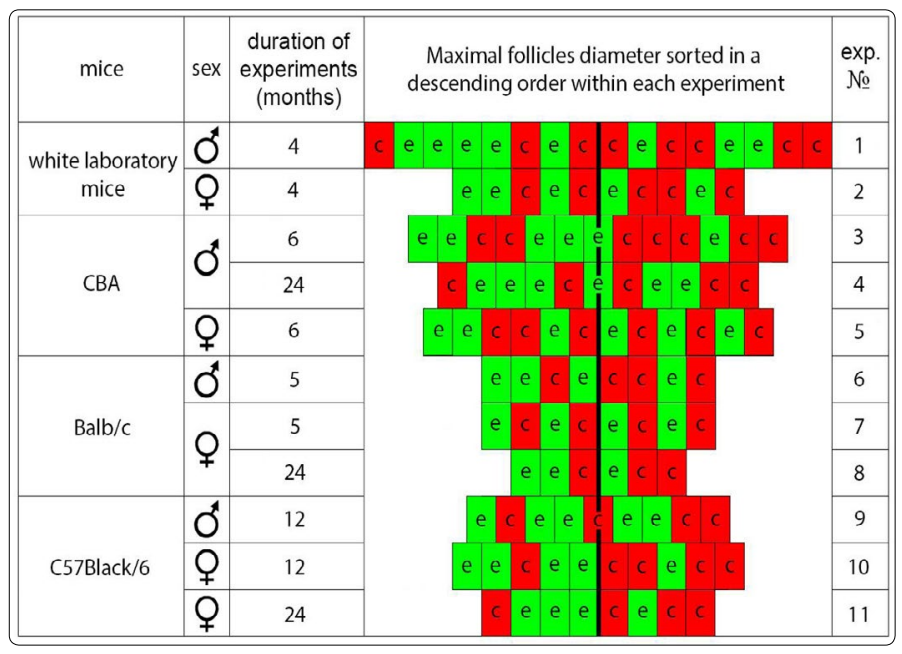

Figure 3. RSHG influence on hair follicles size. e - experimental, c - control.

As one can see, there is a tendency of follicle size increase in each of eleven experiments. Experimental follicles are located more left-of-center in the rows than controls. As it occurs in eleven independent experiments, the probability of a mistake is less than $0,001\left(1 / 2^{11}\right)$.

\section{Long term RSHG application increases volumes of hair roots}

To have additional evidence of the effect of RSHG on follicle size we performed measurements of the volumes of plucked hair roots (fig. 4a). We measured roots from 5 control and 5 experimental mice (25-30 hairs from each) and made ascending distributions (fig $4 \mathrm{c}$ and $4 \mathrm{~d}$ ).

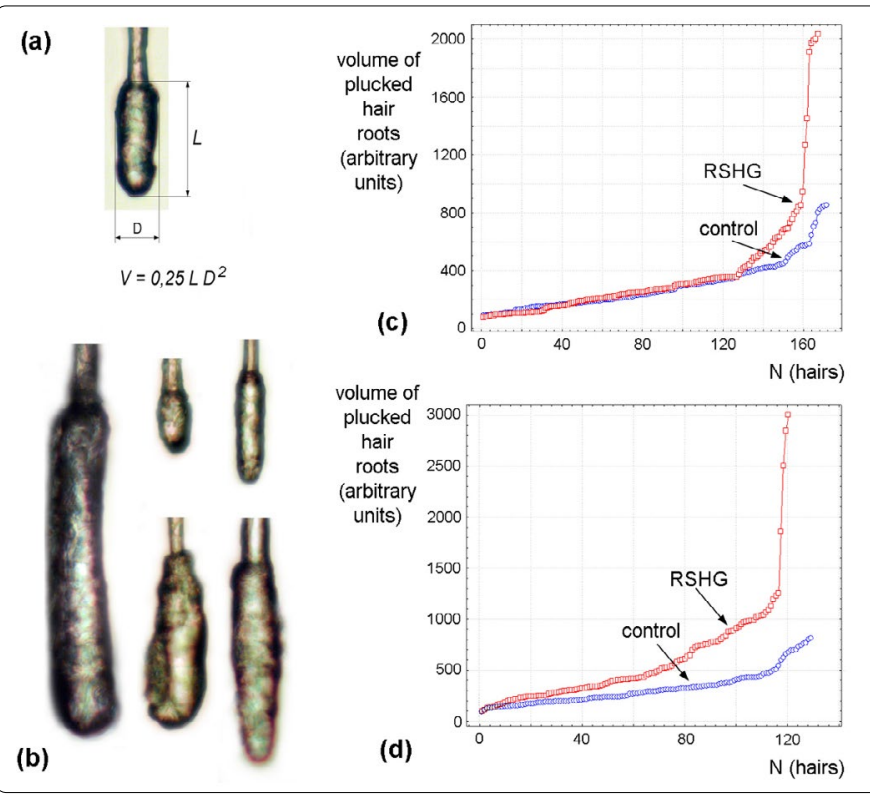

Figure 4. Influence of RSHG on hair roots volume distribution

(a) Scheme of root volume calculation, (b) - examples of various roots, (c) results of 130 days RSHG application to females of outbred white laboratory mice, (d) results of 90 days of RSHG application to CBA males.

Figures $4 c$ and $4 d$ show the significant $(p<0,01)$ increase of hair roots volumes after RSHG application. At the same time, it is clear that volumes of the smallest hair roots are not affected by RSHG. Instead, large hair root are sensitive to RSHG. Especially responsive are a few percent of hairs with the largest root volumes, which increased 2.5 - 3.75 times.

\section{Long term RSHG application increases skin thickness}

The increase of the mean follicle size without affecting the sizes of smallest follicles can indicate that RSHG increases the portion of anagen hairs. So far as in mice there are growth waves of hairs which are matched with the increasing of skin thickness [2] we compare the skin thickness as well.

Fig. 5 represents the results of these experiments. They are very similar to the follicle sizes results. Each of ten experiments showed a tendency to the increase of skin thickness under the influence of RSHG. The probability of a mistake was less than $0,001\left(1 / 2^{10}\right)$.

\begin{tabular}{|c|c|c|c|c|c|c|c|c|c|c|c|c|}
\hline mice & sex & $\begin{array}{l}\text { duration of } \\
\text { experiments } \\
\text { (months) }\end{array}$ & \multicolumn{9}{|c|}{$\begin{array}{l}\text { Skin thickness sorted in a descending } \\
\text { order within each experiment }\end{array}$} & \multirow{2}{*}{\begin{tabular}{|c|}
$\begin{array}{c}\text { exp. } \\
\text { № }\end{array}$ \\
1
\end{tabular}} \\
\hline \multirow{2}{*}{$\begin{array}{l}\text { white laboratory } \\
\text { mice }\end{array}$} & $0^{\prime}$ & 4 & e e & e & & e & c & c & e $\epsilon$ & e c e & c $\mathrm{C}$ & \\
\hline & Q & 4 & & & & e & c & & c & C $\mathrm{C}$ & & 2 \\
\hline \multirow{3}{*}{ CBA } & & 6 & e & e & & $\mathrm{e}$ & e & c & e $\epsilon$ & e $c$ & C $\mathrm{C}$ & 3 \\
\hline & & 24 & & e & e & c & e & e & c & e $c|c| c \mid$ & C & 4 \\
\hline & q & 6 & & e & e & c & e & e & c & 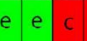 & c & 5 \\
\hline \multirow{3}{*}{$\mathrm{Balb} / \mathrm{c}$} & $0^{1}$ & 5 & & & e & e & e & c & c 6 & c $c \mid c$ & & 6 \\
\hline & \multirow{2}{*}{$q$} & 5 & & & e & c & c & e & e & e c & & 7 \\
\hline & & 24 & & & & e & e & & c & e c & & 8 \\
\hline \multirow{2}{*}{ C57Black/6 } & $\delta^{\prime}$ & 12 & & & e & e & c & e & c & e & & 9 \\
\hline & Q & 12 & & & & e & e & & e & e & & 10 \\
\hline
\end{tabular}

Figure 5. RSHG influence on skin thickness. e - experimental, c - control. 
Some of the results are presented in fig. 6. Histological analysis was performed after different periods of RSHG applications (from 4 to 24 months). We found that RSHG treatment did not abolish the waves of hair growth (fig 6a). Alterations of skin thickness were mainly due to the variability of the thickness of adipose.

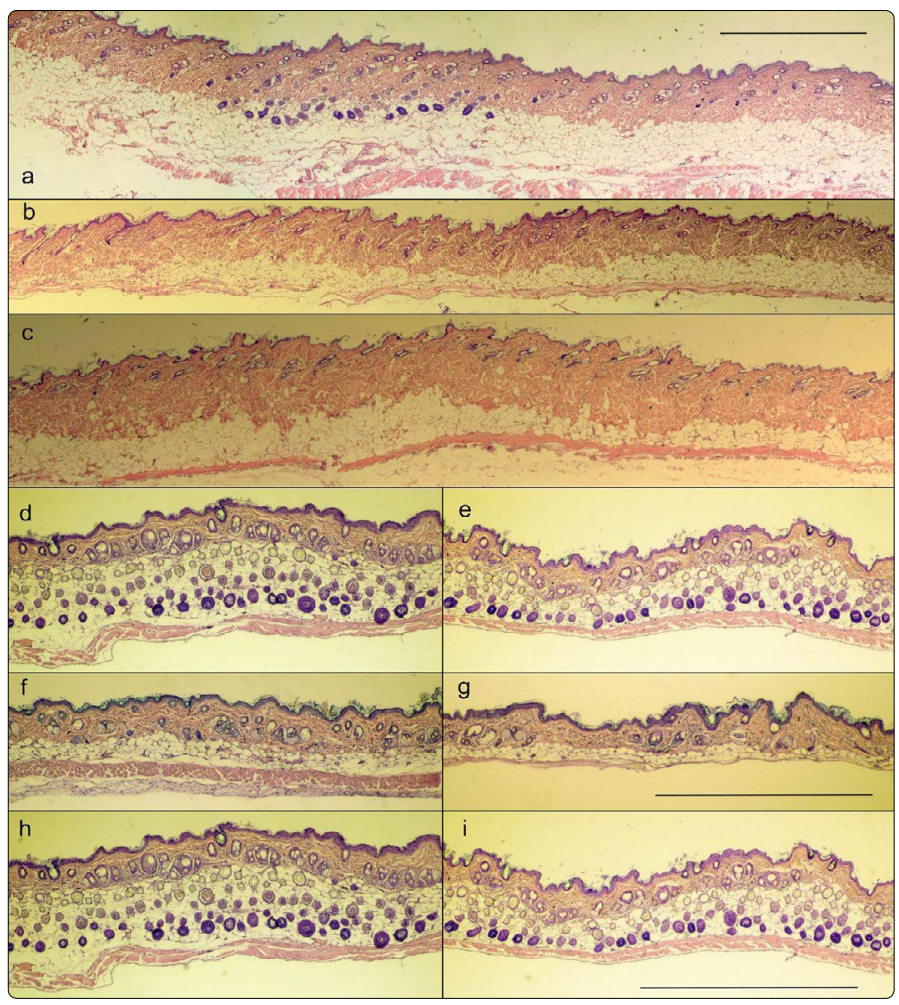

Figure 6. RSHG influence on mice skin thickness (a-c) CBA males, the rest - Balb/c females. (a) and (c) - 6 months RSHG application, (b) - control. RSHG application does not abolish hair growth waves (a). (d) and (f) - 12 months RSHG application, (e) and (g) - control. (h) - 24 months RSHG application, (i) - control. Bars $1 \mathrm{~mm}$. Groups of photos $(\mathrm{a}-\mathrm{c}),(\mathrm{d}-\mathrm{g})$ and $(\mathrm{h}-\mathrm{i})$ have the same magnifications. Hematoxylin-eosin staining, digital contrast.

\section{RSHG application stimulates overgrowth of bald areas of old mice skin}

We wanted to find a clear manifestation of RSHG action in animals. Young mice have good hair, so it is difficult to strongly stimulate their growth. We decided to work with old black mice, which exhibit clear defects of hair growth. In a considerable population (around one hundred) of more than two years old C57Black/6 mice we choose two groups of five mice with visible alopecia areas. After two months of RSHG application, alopecia regions were overgrown by hair in both groups (fig. 7).

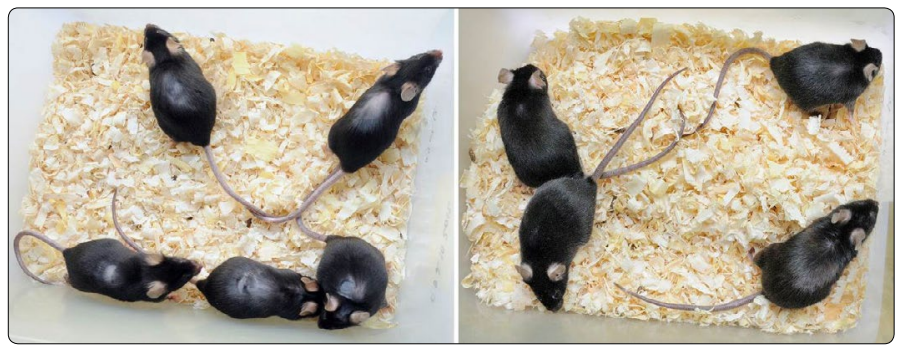

Figure 7. Decrease of bald areas on the skin of senescent C57Black/6 mice after 2 months of RSHG application. Left - before application, right - after application.
Transcriptome analysis is a modern investigation technique that permits to entirely cover alterations of cellular gene expression. We chose to use it in the process of RSHG investigation because due to the complex composition RSHG can simultaneously act in different directions.

At present, we do not know that cells are the target of RSHG in vivo. Those may be hair keratinocytes, dermal papilla cells, dermal fibroblasts, cells of immune system, nerve cells etc. Selective and rapid isolation of these cells from murine tissues without changing their pattern of gene expression is at present impossible.

Thus, we have selected as a model the culture of adult human skin fibroblasts. This culture (strain 1608) was comprehensively investigated [24]. Proliferative capabilities of cells allow working with a homogeneous cell population where $90 \%$ cells are in cell cycle.

The transcriptome analysis using the MetaCore program showed with a high reliability that among most significant processes enriched in differentially expressed genes (Pathway Maps) was cell cycle, cytoskeleton dynamics, epithelial-tomesenchymal transition (EMT), cell adhesion and extracellular matrix remodeling (fig. 8).

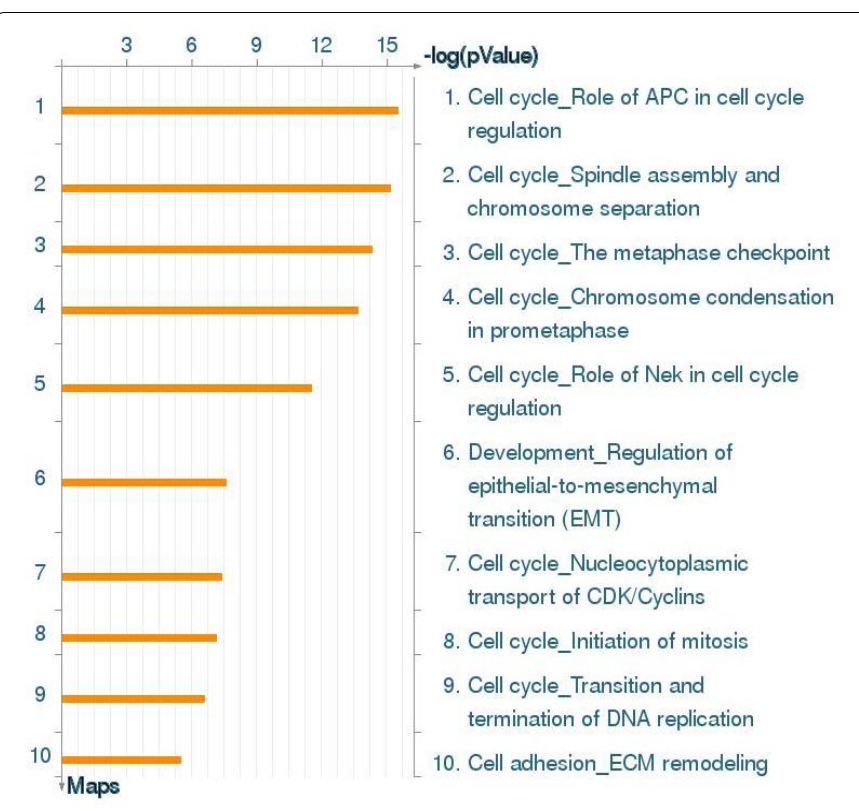

Figure 8. Pathway Maps under the influence of RSHG.

Networks analysis (ProcessNetworks) revealed that most significant changes are observed in cytoskeleton (-log $(p$ Value $)=23)$, cell cycle $(-\log (p V a l u e)=22)$, cell adhesion and cell matrix $(-\log (p$ Value $)=8)$.

Metabolic Networks analysis revealed activation of the $\mathrm{N}$-acyl-sphingosine phosphate and sphingomyelin pathways with [-log (pValue) = 7], (fig. 9). 


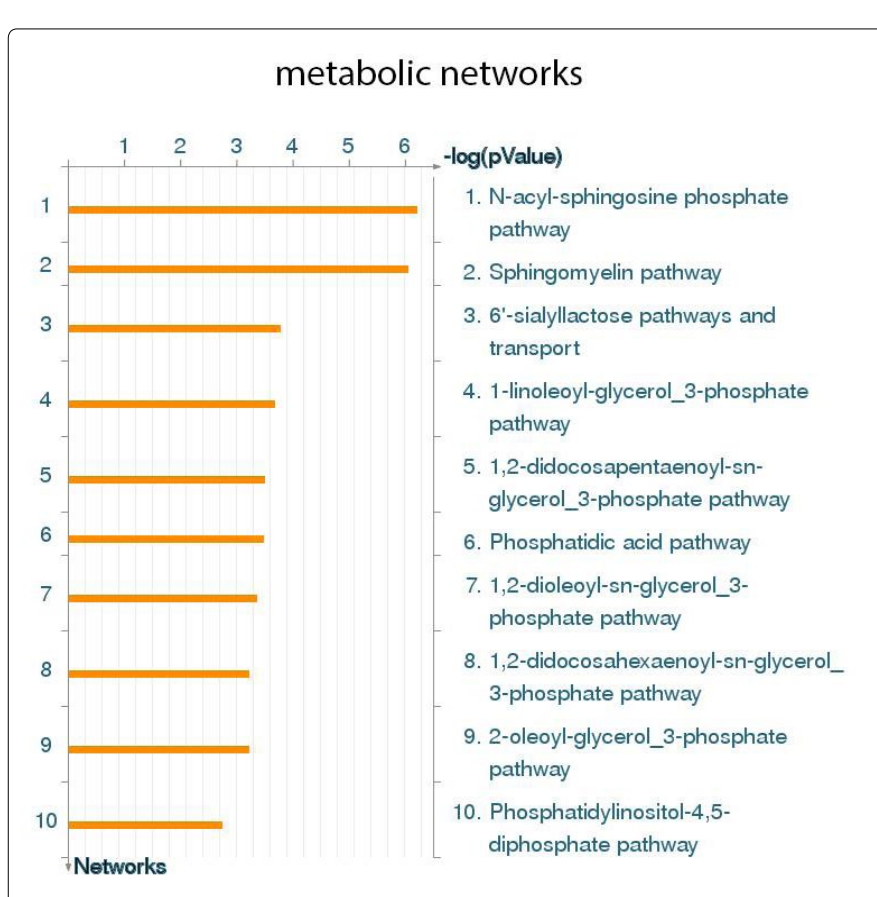

Figure 9. Changes in metabolic networks under the influence of RSHG.

Gene Ontology Processes analysis pointed again to the cell cycle and various developmental processes.

\section{Discussion}

During the primary study, we found that biggest follicles were observed in RSHG treated specimens. In the course of quantitative analysis, we confirmed that indeed RSHG treatment results in the increase of follicle size (fig. 2-3). There are several types of hairs on the back of the mouse. A few percents of the largest hairs constitute guard hairs. These hairs are also known as tylotrich hairs and they exhibit particular mechanisms and timing of embryonic development. Unlike other, hairs they have a connection with the nervous system to perform a sensory function [25]. On histological sections, guard hair follicles can be identified by their exceptional size. Probably the RSHG affects guard hairs especially strongly.

The second approach was the investigation of plucked hair roots. The plucked hair shaft has been used in medical research over the last 60 years [26]. Gho and colleagues demonstrated that most of the epithelial structures from the hair follicle remain attached to the plucked hair [27]. The maintenance of integrity of the outer root sheet after hair plucking has been documented by Limat and colleagues [28]. The outer root sheet is visible as a white layer covering the root [29]. Hair follicle stem cells lie in the bulge of the outer root sheet and give rise to keratinocytes and other cell types.

Because the size of the follicle is determined by cell number, we chose the hair root volume as the best parameter to characterize the follicle size. To simplify the task, we calculated the volumes circumscribed around root cylinders. Results obtained with this approach were similar to the results obtained by studying histological sections but much more reliable. Moreover this approach provided hair roots volumes distribution that showed that RSHG did not change the sizes of the smallest (resting) roots (fig. $4 \mathrm{c}$ and d), but increased the sizes of the biggest ones.

Mice hair coat contains a mix of four different types of hair: guard, awl, auchene and zigzag, as well as specialized hairs such as vibrissae and tail hair [25]. Guard hairs are the largest and constitute 1-3\% [25]. As it can be seen from fig. 4, the experimental curves have sharp bends that separate $4.7 \%$ (fig. 4c) and $4.2 \%$ (fig. 4d) of the biggest hairs. Probably the most prominent action of RSHG is directed to guard hairs.

Although the maximal effect was observed in guard hairs, RSHG action was not restricted to them. As it can be seen from fig. 4, a weaker action can overlap about $25 \%$ of the hairs in case of fig. $4 \mathrm{c}$ and most of the hairs in fig $4 \mathrm{~d}$. This observation is consistent with the suggestion that RSHG increases the ratio of anagen hairs.

Baldness development is typically due to follicles miniaturization. So the follicles enlargement under the influence of RSHG can be considered as an anti-balding action.

Well-developed adipose is a necessary precondition for hair growth. At the beginning of the growth, hair follicles increase their volumes through cell multiplication and immerse deeply into adipose. In animals with coordinated hair replacement the overall skin thickness increases in response to massive anagen initiation at the expense of subcutis elaboration [2]. There are naturally determined waves of hair growth during mice development. The ordering of these waves is very regular initially after birth. With passing time they breakdown into "islands of growth", especially on the dorsum [30]. First, we observed that RSHG application does not abolish hair growth waves. Second, we found the increase of skin thickness (Fig. 6), and also detected a significant alteration of subcutis after RSHG treatment (fig. 6).

Previously we showed that RSHG possesses similar activity in humans [21]. We revealed substantial restoration of hair growth, augmentation of subcutis and an overall skin structure normalization on the basis of histological examination of scalps of two volunteers after course of RSHG treatment. It has also been shown that RSHG induces adipogenic differentiation of mesenchymal stromal cells in culture [21]. Based on these results, we suggest that RSHG stimulates adipose elaboration.

Increases of follicle size and of skin thickness are indirect signs of hair growth stimulation. Experiments with aging mice showed that RSHG indeed stimulates hair growth.

To understand the possible mechanisms of RSHG action, we performed transcriptome studies. Analysis reveals some alterations of cell cycle, processes of development, stimulation of epithelial-to-mesenchymal transition (EMT), extracellular matrix (ECM) remodeling and lipid metabolism pathways. Considering that the RSHG mainly consists of lipid derivatives (Fig. 1), it is clear that it must change the metabolism of lipids. Activation of EMT in case of fibroblasts means increase of motility and cytoskeleton alterations. ECM remodeling and changes of cells adhesion are also connected to EMT.

Observed alterations of cell cycle gene expression suggest that RSHG action leads to accumulation of cells in the G2 period. 
Such accumulation is typical for keratinocytes [31]. This result was not unexpected, because previously we have shown that RSHG does not stimulate proliferation of various cells in vitro [22].

Obtained results together with earlier ones allow us to propose hypothesis about mechanisms of RSHG action. Previously we demonstrated that RSHG increases cell death under conditions of oxidative stress [22]. Oxidative stress is known to induce cell damage and senescence in vivo. Selective elimination of senescent cells in the body decreases senescence associated pathology [32]. Probably the same situation takes place in hair follicles: decreasing senescent cells ratio has an anti-aging effect.

After application to the skin, RSHG needs to overcome the layer of cornified keratinocytes to realize its action. Another way is transfollicular transport [33]. There is a gap around hair and any preparation including nanoparticles [34] can easily achieve follicular region. Thus, maximal delivery of RSHG should be addressed to follicle cells. If there are senescent or damaged cells within the follicle, RSHG could induce their elimination that should stimulate regeneration process and hair growth.

Apparently the main locale of cell aging in skin is connective tissue including dermal papilla and follicles envelop. Indeed, connective tissue develops aging markers more rapidly than epithelial tissue [35]. Dermal papilla is the principal regulator of hair growth [36]. Moreover, it was shown that hair stem cells (epithelial cells) are stored in the skin even after fully developed baldness [37].

During aging, the decrease of stem cell functions is determined not only by alteration of stem cells per se but also by aging of stem cell niche. Thus, the function of hematopoietic stem cells depends on stromal cells of bone marrow [38]; the function of myogenic stem cells depends on myogenic niche [39].

Considering a hair follicle as a system composed of two components, connective tissue and epithelium, one can notice a principal difference of their lifetime. Epithelial cells actively proliferate during each hair cycle and the new groups of stem cells go from the bulge to maintain new rounds of proliferation. At the same time, dermal papilla growth is restricted and their cells should develop senescent phenotype relatively rapidly as any slowly dividing or non-dividing cell.

We suggest that RSHG action is not restricted to the stimulation of senescent cells elimination. Previously we have shown that RSHG induce autophagy [22]. Mild autophagy exhibits anti-aging activity [40]. It cleans cells from damaged proteins and organelles, hastens their turnover and therefore improves cells functions. Autophagy induction is capable to preserve stem cells and inhibit the development of baldness in mice with increased expression of Wnt1 [41].

On the other hand, autophagy induction leads to production of high energy compounds that hasten cell metabolism, as it happens with autophagy induction in tumorassociated fibroblasts [42]. Such processes can support both hair growth and subcutis elaboration, a precondition of anagen onset. However, excessive autophagy can increase cell death [43], and, probably, senescent and damaged cells are more sensitive to it than healthy cells.

\section{Conclusions}

We suggest that a possible mechanism of action of RSHG can be selective elimination of senescent cells in hair follicles. Such preparation as RSHG should stimulate the hair growth in various kind of alopecia. The molecular mechanisms of RSHG action require further investigation.

\section{Acknowledgements}

The authors thank GZ Laboratories (UK) Ltd for providing of "Satura ${ }^{\circledR}$ Rosta" balsam and its basis. We are gratefull to Dr. S.M. Terekhov for providing 1608 cells. We thank Dr. G.V. Zigmund for helpful discussion and Dr. I. Prudovsky (Maine Medical Center Research Institute, USA) for critical reading of the manuscript. Some of the experiments were performed using equipment of the GENOM Collective Access Center of the Engelhardt Institute of Molecular Biology. This work was supported by the Russian Federation Ministry of Education and Science (agreement no. 8127)

\section{Conflict of Interest}

No conflicts of interest have been declared.

\section{References}

1. Stenn KS, Paus R. Controls of hair follicle cycling. Physiol Rev. 2001; 81(4): 449-494. doi: 10.1152/physrev.2001.81.1.449

2. Plikus MV, Chuong CM. Complex hair cycle domain patterns and regenerative hair waves in living rodents. J Invest Dermatol. 2008; 128(5): 1071-1080. doi: 10.1038/sj.jid.5701180

3. Fossel M. Cells, Aging, and Human Disease. 2004; Oxford University Press. ISBN 0195140354.

4. de Lacharrie 're O, Deloche $C$, Misciali $C$, et al. Hair diameter diversity: a clinical sign reflecting the follicle miniaturization. Arch Dermatol. 2001; 137(5): 641-646.

5. Sinclair RD, Banfield CC, Dawber PR. Handbook of Diseases of the Hair and Scalp. Oxford, England, Blackwell Science Ltd. 1999; 240: ISBN 0-86542-928-926.

6. Hamilton J. Male hormone is prerequisite and incitant in common baldness. Amer J Anat. 1942; 71(3): 451-480. doi: 10.1002/aja.1000710306

7. Inui S, Fukuzato Y, Nakajima T, Yoshikawa K, Itami S. Androgen-inducible TGFbeta1 from balding dermal papilla cells inhibits epithelial cell growth: a clue to understand paradoxical effects of androgen on human hair growth. FASEB J. 2002; 16(14): 1967-1969. doi: 10.1096/fj.02-0043fje

8. Inui S, Fukuzato Y, Nakajima T, Yoshikawa K, Itami S. Identification of androgen-inducible TGF-beta1 derived from dermal papilla cells as a key mediator in androgenetic alopecia. J Investig Dermatol. Symp. Proc. 2003; 8(1): 69-71. doi: 10.1046/j.1523-1747.2003.12174.x

9. Hibino T, Nishiyama T. Role of TGF-beta2 in the human hair cycle. J Dermatol Sci. 2004; 35(1): 9-18. doi: 10.1016/j.jdermsci.2003.12.003

10. Kwack MH, Sung YK, Chung EJ, et al. Dihydrotestosterone-inducible dickkopf 1 from balding dermal papilla cells causes apoptosis in follicular keratinocytes. J Invest Dermatol. 2008; 128(2): 262-269. doi: 10.1038/ sj.jid.5700999

11. Inui S, Itami S. Molecular basis of androgenetic alopecia: from androgen to paracrine mediators through dermal papilla. J Dermatol Sci. 2011; 61(1): 1-6. doi: 10.1016/j.jdermsci.2010.10.015 
12. Messenger AG, Rundegren J. Minoxidil: mechanisms of action on hair growth. Br J Dermatol. 2004; 150(2): 186-194.

13. Olsen EA, Dunlap FE, Funicella $T$, et al. A randomized clinical trial of $5 \%$ topical minoxidil versus $2 \%$ topical minoxidil and placebo in the treatment of androgenetic alopecia in men. J Amer Acad Dermatol. 2002; 47(3): 377385 .

14. Price VH, Menefee $E$, Strauss PC. Changes in hair weight and hair count in men with androgenetic alopecia, after application of $5 \%$ and $2 \%$ topical minoxidil, placebo, or no treatment. J Am Acad Dermatol. 1999; 41(5, pt 1): 717-721.

15. Drake $L$, Hordinsky $M$, Fiedler $V$, et al. The effects of finasteride on scalp skin and serum androgen levels in men with androgenetic alopecia. J Amer Acad Dermatol. 1999; 41(4): 550-554.

16. Whiting DA, Waldstreicher J, Sanchez M, Kaufman KD. Measuring reversal of hair miniaturization in androgenetic alopecia by follicular counts in horizontal sections of serial scalp biopsies: results of finasteride $1 \mathrm{mg}$ treatment of men and postmenopausal women. J Investig Dermatol Symp Proc. 1999; 4(3): 282-284.

17. Finasteride Male Pattern Hair Loss Study Group. Long-term (5-year) multinational experience with finasteride $1 \mathrm{mg}$ in the treatment of men with androgenetic alopecia. Eur J Dermatol. 2002; 12(1): 38-49.

18. Price $\mathrm{VH}$, Roberts $J \mathrm{~L}$, Hordinsky $\mathrm{M}$, et al. Lack of efficacy of finasteride in postmenopausal women with androgenetic alopecia. J Am Acad Dermatol. 2000; 43 (5 Pt 1): 768-776. doi: 10.1067/mjd.2000.107953

19. Camacho-Martínez FM. Hair loss in women. Semin Cutan Med Surg. 2009; 28(1): 19-32. doi: 10.1016/j.sder.2009.01.001

20. Scheinfeld N. A review of hormonal therapy for female pattern (androgenic) alopecia. Dermatol Online J. 2008; 14(3): 1.

21. Vishnyakova KS, Rozinova VN, Yegorov YE. Satura Rosta preparation restores hair growth and promotes adipogenic differentiation of mesenchymal stem cells. International J Innovations Bio-Sci. 2013; 3(2): 1016.

22. Vishnyakova KS, Popov KV, Vorotelyak EA, Faizullin RR, Artyukhov S, Yegorov $Y$ E. Possible role of autophagy activation in stimulation of regeneration. $\mathrm{Mol}$ Biol. 2013; 47(5): 692-700. doi: 10.1134/S002689331305021X

23. Vishnyakova KS, Babizhayev MA, Aliper AM, Buzdin AA, Kudryavzeva AV, Yegorov YE. Stimulation of Cell Proliferation by Carnosine: Cell and Transcriptome Approaches. Mol Biol. 2014; 48 (5): 718-726. doi: 10.1134/ S0026893314050161

24. Yegorov $\mathrm{YE}$, Moldaver MV, Vishnyakova KS, et al. Enhanced control of proliferation in Telomerized cells. Ontogenez. 2007; 38(2): 105-119.

25. Duverger O, Morasso MI. Epidermal patterning and induction of different hair types during mouse embryonic development. Birth Defects Res C Embryo Today. 2009; 87(3): 263-272. doi: 10.1002/bdrc.20158

26. Schembri K, Scerri C, Ayers D. Plucked Human Hair Shafts and Biomolecular Medical Research. Sci World J. 2013; ID 62053. doi: 10.1155/2013/620531

27. Gho CG, Braun JE, Tilli CM, Neumann HA, Ramaekers FC. Human follicular stem cells: their presence in plucked hair and follicular cell culture. $\mathrm{Br} J$ Dermatol. 2004; 150(5): 860-868. doi: 10.1111/j.0007-0963.2004.05862.x
28. Limat A, French LE, Blal L, Saurat J, Hunziker T, Salomon D. Organotypic cultures of autologous hair follicle keratinocytes for the treatment of recurrent leg ulcers. J Amer Acad Dermatol. 2003; 48(2): 207-214. doi: $10.1067 / \mathrm{mjd} .2003 .69$

29. Raab S, Klingenstein M, Liebau S, Linta L. A Comparative View on Human Somatic Cell Sources for iPSC Generation. Stem Cells Inter. 2014; ID 768391: 12. doi: 10.1155/2014/768391

30. Chase HB, Eaton GJ. The growth of hair follicles in waves. Ann. NY Acad. Sci. 1959; 83: 365-368. DOI: 10.1111/j.1749-6632.1960.tb40912.x

31. Gandarillas A, Davies D, Blanchard JM. Normal and c-Myc-promoted human keratinocyte differentiation both occur via a novel cell cycle involving cellular growth and endoreplication. Oncogene. 2000; 19(29): 3278-3289. doi: 10.1038/sj.onc. 1203630

32. Baker DJ, Wijshake T, Tchkonia T, et al. Clearance of p16lnk4a-positive senescent cells delays ageing-associated disorders. Nature. 2011; 479: 232-236. doi: 10.1038/nature10600

33. Knorr F, Lademann J, Patzelt A, Sterry W, Blume-Peytavi U, Vogt A. Follicular transport route-research progress and future perspectives. Eur J Pharmac Biopharmac. 2009; 71(2): 173-180. doi: 10.1016/j.ejpb.2008.11.001

34. Vogt A, Combadiere B, Hadam S, Stieler KM, Lademann J, Schaefer HB. 40 $\mathrm{nm}$, but not 750 or $1,500 \mathrm{~nm}$, nanoparticles enter epidermal CD1a+ cells after transcutaneous application on human skin. J Investig Dermatol. 2006; 126(6): 1316-1322. doi: 10.1038/sj.jid.5700226

35. Stern MM, Bickenbach JR. Epidermal stem cells are resistant to cellular aging.AgingCell.2007;6(4):439-452.doi:10.1111/j.1474-9726.2007.00318.x

36. Schmidt-Ullrich $R$, Paus R. Molecular principles of hair follicle induction and morphogenesis. Bioessays. 2005; 27(3): 247-261. doi: 10.1002/ bies.20184

37. Garza LA, Yang CC, Zhao T, et al. Bald scalp in men with androgenetic alopecia retains hair follicle stem cells but lacks CD200-rich and CD34positive hair follicle progenitor cells. J Clin Invest. 2011; 121(2): 613-622. doi: $10.1172 / \mathrm{JCl} 44478$

38. Ju $Z$, Jiang $H$, Jaworski $M$, et al. Telomere dysfunction induces environmental alterations limiting hematopoietic stem cell function and engraftment. Nat. Med. 2007; 13(6): 742-747 doi: 10.1038/nm1578

39. Carlson M E, Conboy IM. Loss of stem cell regenerative capacity within aged niches. Aging Cell. 2007; 6(3): 371-382. doi: 10.1111/j.1474-9726.2007.00286.x

40. Rubinsztein DC, Marino G, Kroemer G. Autophagy and aging. Cell. 2011; 146(5): 682-695 doi: 10.1016/j.cell.2011.07.030

41. Castilho RM, Squarize CH, Chodosh LA, Williams BO, Gutkind JS. mTOR mediates Wnt induced epidermal stem cell exhaustion and aging. Cell Stem Cell. 2009; 5(3): 279-289. DOI: 10.1016/j.stem.2009.06.017

42. Capparelli C, Guido C, Whitaker-Menezes D, et al. Autophagy and senescence in cancer-associated fibroblasts metabolically supports tumor growth and metastasis via glycolysis and ketone production. Cell Cycle. 2012; 11(12): 2285-2302. doi: 10.4161/cc.20718

43. Sharma K, Le N, Alotaibi M, Gewirtz D A. Cytotoxic autophagy in cancer therapy. Int J Mol Sci. 2014; 15(6): 10034-10051. doi: 10.3390/ijms150610034 